\title{
Homing commitment of lymphocytes activated in the human gastric and intestinal mucosa
}

\author{
M Quiding-Järbrink, I Ahlstedt, C Lindholm, E-L Johansson, H Lönroth
}

\begin{abstract}
Background-Gastric infection with the human pathogen Helicobacter pylori results in a large accumulation of $\operatorname{IgA}$ and IgM secreting cells in the gastric mucosa. The molecular mechanisms resulting in $B$ cell migration to the gastric mucosa in $H$ pylori infection are however not known.

Aims-To examine expression of the mucosal homing receptor integrin $\alpha 4 \beta 7$ and the homing receptor for secondary lymphoid tissues, L-selectin, on lymphocytes activated by gastric, intestinal, or systemic antigens. Furthermore, to examine gastric expression of the mucosal addressin cellular adhesion molecule 1 (MAdCAM-1), the endothelial counterreceptor to integrin $\alpha 4 \beta 7$.
\end{abstract}

Subjects and methods-H pylori infected individuals were immunised by either gastric $(n=8)$ or intestinal $(n=8)$ delivery of an inactivated cholera vaccine. The resulting circulating vaccine specific $B$ cells were sorted according to $\alpha 4 \beta 7$ and L-selectin expression and assayed for production of IgA and IgG using an enzyme linked immunospot assay. In addition, circulating CD4+ T cells from seven $H$ pylori infected individuals were fractionated according to $\alpha 4 \beta 7$ and $L-$ selectin expression. The resulting $T$ cell fractions were then assayed for specific proliferation against $H$ pylori or the systemic antigen tetanus toxoid. Finally, gastric expression of MAdCAM-1 was determined by immunohistochemistry in $\mathrm{H}$ pylori infected $(n=16)$ and uninfected $(n=8)$ individuals.

Results-Virtually all B cells induced by both gastric and intestinal antigen delivery expressed $\alpha 4 \beta 7$ whereas less then half coexpressed L-selectin. Furthermore, $\boldsymbol{H}$ pylori reactive $T$ cells were mainly found in the $\alpha 4 \beta 7+\mathrm{L}-$ selectin $+T$ cell fraction whereas tetanus specific $T$ cells were largely $\alpha 4 \beta 7-\mathrm{L}-$ selectin+. MAdCAM-1 was present in similar amounts in gastric mucosa from $H$ pylori infected and uninfected individuals.

Conclusions-B cells and $T$ cells activated by antigens delivered to the gastric mucosa express the mucosal homing receptor integrin $\alpha 4 \beta 7$, as do cells activated in the intestine. Together with the observation that gastric endothelial cells express MAdCAM-1, this may partly explain the homing of lymphocytes activated in the stomach or in the small intestine to the gastric mucosa.

(Gut 2001;49:519-525)
Keywords: lymphocyte trafficking; integrin $\alpha 4 \beta 7$; L-selectin; stomach; Helicobacter pylori

Helicobacter pylori infection of the human stomach results in active chronic gastritis in almost all infected subjects, with a characteristic infiltration of large numbers of lymphocytes and neutrophils, and formation of organised lymphoid follicles with discrete $\mathrm{B}$ and $\mathrm{T}$ cell areas. ${ }^{1-3}$ In a previous study, we also noted a very large increase in the frequencies of $\operatorname{IgA}$ and IgM secreting cells in the gastric mucosa of $H$ pylori infected individuals compared with uninfected subjects. ${ }^{4}$ Furthermore, following mucosal immunisations with an inactivated cholera vaccine, vaccine specific IgA responses could only be detected in the gastric mucosa of $H$ pylori infected subjects and not in uninfected subjects, even though both groups of volunteers had similar frequencies of IgA secreting cells in the intestinal mucosa. ${ }^{5}$ Taken together, these observations strongly indicate that $H$ pylori infection increases the capacity of the gastric mucosa to recruit lymphocytes.

Tissue recruitment of leucocytes from the circulation is regarded as a multistep process involving several distinct phases. Loose interactions between integrins and their carbohydrate ligands result in the characteristic rolling along the vessel wall and this, together with chemoattractant signals from the tissue, activate integrins to confer strong binding to their endothelial ligands and arrest of the cell, followed by flattening and extravasation. ${ }^{67}$ Presumably, this process can be controlled at any of these steps. Thus far, expression of adhesion molecules by the migrating leucocytes and by endothelial cells in their target organs has received most of the attention, and some of the tissue specific lymphocyte trafficking can be explained by expression of certain adhesion molecules. Thus homing to secondary lymphoid tissues is dependent on expression of the peripheral lymph node addressin (PNAd) on the endothelium which binds to L-selectin expressed on circulating cells. In addition, mucosal endothelium in the intestinal tract express the mucosal addressin cellular adhesion molecule 1 (MAdCAM-1) which binds to integrin $\alpha 4 \beta 7$ on circulating lymphocytes. ${ }^{8}$ Circulating $\mathrm{B}$ and $\mathrm{T}$ cells induced

Abbreviations used in this paper: ASC, antibody secreting cell; ELISPOT, enzyme linked immunospot assay; CTB, cholera toxin B subunit; MAdCAM-1, mucosal addressin cellular adhesion molecule 1 ; MNC, mononuclear cells; MP, membrane protein; PNAd, peripheral lymph node addressin; TT, tetanus toxoid; PBS, phosphate buffered saline; SI, stimulation index; cpm, counts per minute. 
by intestinal immunisation or infection all carry $\alpha 4 \beta 7$ whereas L-selectin is only expressed on a minority of such cells. ${ }^{9-13}$ Recently, it has also been revealed that circulating lymphocytes with different homing commitments respond differently to chemokines ${ }^{14}$ and this is probably an important component of tissue specific lymphocyte migration.

The homing receptor usage of cells homing to gastric tissues is however still unknown. It has recently been demonstrated that the venules in the human stomach mucosa express MAdCAM- $1,{ }^{16}$ and the lymphoid follicles that develop in response to $H$ pylori infection have endothelial cells expressing both PNAd and MAdCAM..$^{16}{ }^{17}$ To what extent this expression correlates with recruitment of circulating lymphocytes has not been examined. To better define the molecular recognition events resulting in lymphocyte trafficking to the $H$ pylori infected gastric mucosa, we have taken several different approaches. Homing receptor expression on B cells induced by gastric immunisation was determined by immunomagnetic cell sorting followed by enzyme linked immunospot assay (ELISPOT) assays. Expression of homing receptors by $H$ pylori specific circulating $\mathrm{CD} 4+\mathrm{T}$ cells was also examined by magnetic sorting followed by in vitro stimulation. In addition, expression of MAdCAM-1 on gastric endothelial cells from $H$ pylori infected and uninfected individuals was examined using immunohistochemistry.

\section{Material and methods}

VOLUNTEERS AND IMMUNISATIONS Volunteers

This study was performed following approval from the human research ethics committee of the Medical Faculty, Göteborg University, and all volunteers gave informed consent to participate. Twenty three $H$ pylori infected individuals were recruited among patients attending the gastroenterology unit at Sahlgrenska University Hospital, Göteborg, or by serological screening of healthy blood donors. ${ }^{18}$ Before enrolment, $H$ pylori infection was demonstrated by positive serology or urea breath test. ${ }^{18}$ None of the infected volunteers had an active ulcer or was on any medication for at least one week preceding the study.

Sixteen $H$ pylor $i$ infected volunteers received two doses of an oral cholera vaccine (Dukoral; SBL Vaccin, Stockholm, Sweden $)^{19}$ two weeks apart. Each dose of the cholera vaccine consisted of $10^{11}$ killed Vibrio cholerae organisms and $1 \mathrm{mg}$ of recombinant cholera toxin $\mathrm{B}$ subunit (CTB), given either on the gastric mucosa or in the small intestine. Eight of the volunteers (aged 36-61 years; six males and two females; four asymptomatic carriers, three with dyspeptic symptoms, and one duodenal ulcer patient) were immunised twice intragastrically, as described in our accompanying paper in this issue of $G u t .{ }^{20}$ Briefly, $4 \mathrm{ml}$ of vaccine were directly distributed as small droplets through the endoscope over the antral mucosa, with the patient in the left lateral position. Another group of eight volunteers (aged 33-58 years; four males and four females; five asymptomatic carriers, two with dyspeptic symptoms, and one duodenal ulcer patient) received the vaccine in the small intestine as previously described. $^{20} \mathrm{~A}$ gastroduodenoscopy was performed and the endoscope was introduced down to the level of the ligament of Treitzthat is, approximately $30 \mathrm{~cm}$ distal to the pylorus sphincter-and the vaccine was delivered via an endoscope in a total volume of $20 \mathrm{ml}$.

Seven additional $H$ pylori infected volunteers (aged 22-50 years; four males and three females; all asymptomatic carriers) were recruited for studies of peripheral blood $\mathrm{T}$ cell activation. Furthermore, eight healthy volunteers without $H$ pylori infection (aged 22-36 years; five males and three females) were recruited to determine MAdCAM-1 expression in healthy volunteers.

\section{SPECIMEN COLLECTION}

Heparinised venous blood was collected before and one week after the last immunisation from the immunised volunteers, and used for analysis of antibody secreting cells (ASC). On the first occasion, four antral biopsies $2 \mathrm{~mm}$ in diameter and encompassing the epithelium and lamina propria were also collected from each volunteer under local anaesthesia. Gastric biopsies were also collected from the eight uninfected individuals. The biopsies were immediately embedded in OCT compound, frozen in liquid nitrogen, and subsequently used for immunohistochemical detection of MAdCAM-1. One additional biopsy from each volunteer was fixed in formalin, and gastritis was graded from 0 to 3 (none, mild, moderate, or severe), according to the upgraded Sydney system. ${ }^{21}$

Seven $H$ pylori infected volunteers were bled once for collection of venous blood, used for $\mathrm{T}$ cell activation studies.

ISOLATION OF MONONUCLEAR CELLS (MNC)

MNC were isolated from heparinised blood by gradient centrifugation on Ficoll-Hypaque (Pharmacia, Uppsala, Sweden). Interface MNC were collected, washed three times with cold phosphate buffered saline (PBS), and resuspended in cold PBS supplemented with $1 \%(\mathrm{v} / \mathrm{v})$ fetal calf serum or in Iscove's medium with $5 \%$ fetal calf serum and $100 \mu \mathrm{g} / \mathrm{ml}$ of gentamicin (Gibco Europe, Edinburgh, UK) (complete medium). Cell suspensions were kept on ice prior to being further fractionated or assayed for ASC numbers.

DETECTION OF ADHESION MOLECULES ON CIRCULATING ASC

Cell surface expression of integrin $\alpha 4 \beta 7$ and L-selectin by isolated ASC was determined using a combination of immunomagnetic cell sorting and ELISPOT techniques. ${ }^{22}$ Briefly, paramagnetic beads (Dynabeads; Dynal, Oslo, Norway) were coated with monoclonal antibody clone ACT-1 (a kind gift from Dr DJ Ringler, Leukosite Inc., Cambridge, Massachusetts, USA) or Dreg-56 (Pharmingen, San Diego, California, USA), specifying integrin 
$\alpha 4 \beta 7$ and L-selectin, respectively. Coated beads were washed and mixed with the MNC suspensions, and following magnetic separation the resulting positively and negatively selected cell suspensions were resuspended in equal volumes of complete medium. They were then assayed for frequencies of CTB specific IgA and IgG secreting cells and whole $V$ cholerae specific IgA and IgM secreting cells using a two colour ELISPOT technique. ${ }^{45}$ These particular isotypes were chosen for analyses based on our previous studies, showing that CTB induces only weak circulating IgM responses, and that the whole cell component of the vaccine only gives rise to negligible IgG responses. ${ }^{24} 25$

The percentage of ASC expressing a certain marker was calculated by dividing the number of ASC in the positive fraction by the total number of ASC in the positive and negative fractions assayed at the same frequency. Unfractionated cells were assayed in parallel to positively and negatively selected cell suspensions, and the sum of spots in the positive and negative ASC fractions consistently corresponded (within $80-120 \%$ ) to the sum of spots in wells incubated with unfractionated cells.

DETECTION OF ADHESION MOLECULES ON

CIRCULATING T CELLS

Peripheral blood $\mathrm{T}$ cells were prepared from MNC suspensions from seven non-immunised volunteers by overnight incubation with 2-aminoethylisothiouroniumbromide hydrobromide treated sheep red blood cells. CD4+ and CD8+ cells were then isolated from the peripheral blood $\mathrm{T}$ cell suspension by incubation with Dynabeads coated with antibodies to CD4 or CD8, respectively (Dynal). The beads were subsequently removed by incubation with detachabead solution (Dynal) according to the manufacturer's instructions. The remaining non- $T$ cell fraction obtained after SRBC isolation of $\mathrm{T}$ cells consisted of approximately one third B cells (CD19+) and two thirds monocytes (CD14+), as determined by flow cytometry, and was used as a source of accessory cells in T cell stimulations. Further separation according to expression of $\mathrm{L}$-selectin and $\alpha 4 \beta 7$ was performed using the same monoclonal antibodies as before, bound via a DNA linker to Cellection beads from Dynal, according to the manufacturer's instructions. The isolated CD4+ and CD8+ cells were incubated with one of the Cellection bead preparations, and after magnetic separation, the beads were cleaved off using DNAse. This procedure resulted in eight different cell populations: CD4+L-selectin+, CD4+L-selectin-, $\mathrm{CD} 4+\alpha 4 \beta 7+, \quad \mathrm{CD} 4+\alpha 4 \beta 7-, \quad \mathrm{CD} 8+$ L-selectin+, CD $8+$ L-selectin-, CD $8+\alpha 4 \beta 7+$, and CD $8+\alpha 4 \beta 7-$ cells. The magnetically isolated $\mathrm{T}$ cell subpopulations were cultured in triplicate at $1.5 \times 10^{5}$ cells per well together with $1.5 \times 10^{4}$ accessory cells in round bottomed 96 well plates (Nunc, Roskilde, Denmark). The medium used was Iscoves medium supplemented with $5 \%$ human $\mathrm{AB}+$ serum, $3 \mu \mathrm{g} / \mathrm{ml}$ of L-glutamine, and $100 \mu \mathrm{g} / \mathrm{ml}$ of gentamicin. T cell cultures were stimulated with $10 \mu \mathrm{g} / \mathrm{ml}$ of a
$H$ pylori membrane protein (MP) preparation, prepared as previously described, ${ }^{4}$ and pretreated with $1 \mu \mathrm{g} / \mathrm{ml}$ of polymyxin B for one hour to inhibit any residual lipopolysaccharide activity. Parallel cultures were stimulated with $50 \mu \mathrm{g} / \mathrm{ml}$ of tetanus toxoid (TT; a vaccine with which virtually all Swedes have been parenterally immunised several times) or $10 \mu \mathrm{g} / \mathrm{ml}$ of phytohaemaglutinin (Murex Diagnostics Ltd, Temple Hill, UK), which was used as a positive control. T cells were cultured for five days, and $1 \mu \mathrm{Ci}$ of ${ }^{3} \mathrm{H}$ labelled thymidine (Amersham International plc, Little Chalfont, UK) was added to each well during the last 8-14 hours of culture. The amount of incorporated radioactivity was determined in a $\beta$-scintillation counter, and the stimulation index (SI) was calculated by dividing the counts per minute $(\mathrm{cpm})$ value obtained after stimulation with the $\mathrm{cpm}$ value in corresponding cultures without antigen.

IMMUNOHISTOCHEMICAL DETECTION OF

MAdCAM-1

Cryosections (8 $\mu \mathrm{m}$ thick) from antral and duodenal biopsies were mounted on SuperFrost/Plus-glasses (Menzel-Gläzer, Braunschweig, Germany), air dried, and fixed in ice cold acetone for 10 minutes. The sections were incubated with a mouse IgG1 monoclonal antibody specific for human MAdCAM-1 (clone 1AØ3, kindly provided by $\operatorname{Dr} M$ Briskin, Leukosite Inc.), used undiluted, or a von Willebrand factor specific mouse IgG1 monoclonal antibody (Dako, Glostrup, Denmark), diluted 1:25 in PBS for 20 minutes at room temperature. An isotype matched irrelevant mouse antibody (Dako) was included in each experiment as a negative control. After two washings with PBS the sections were incubated with horseradish peroxidase conjugated antimouse IgG (Dako) diluted 1:40 in PBS with $5 \%$ human $\mathrm{AB}$ serum. Bound antibodies were visualised by incubation with 3,3diaminobenzidine (Vector Laboratories Inc., Burlingame, California, USA) for 5-10 minutes at room temperature in the dark. Finally, the sections were counterstained with Mayer's haematoxylin for 10 seconds, dehydrated, and mounted with Mountex (Histolab, Göteborg, Sweden). The entire tissue sections were examined in a Leica microscope at $200 \times$ magnification and the tissue areas were determined with Leica Qwin software (Leica, Germany). Cross sectioned MAdCAM-1 positive and von Willebrand positive vessels were counted and expressed as number of respective vessels per $\mathrm{mm}^{2}$.

STATISTICAL EVALUATIONS

Differences between $\mathrm{T}$ cell subsets were evaluated using the Wilcoxon signed rank test.

\section{Results}

HOMING RECEPTOR EXPRESSION ON B CELLS ACTIVATED BY GASTRIC OR INTESTINAL IMMUNISATION

Expression of L-selectin and integrin $\alpha 4 \beta 7$ was determined on circulating cells induced by gastric or intestinal administration of CTB and 

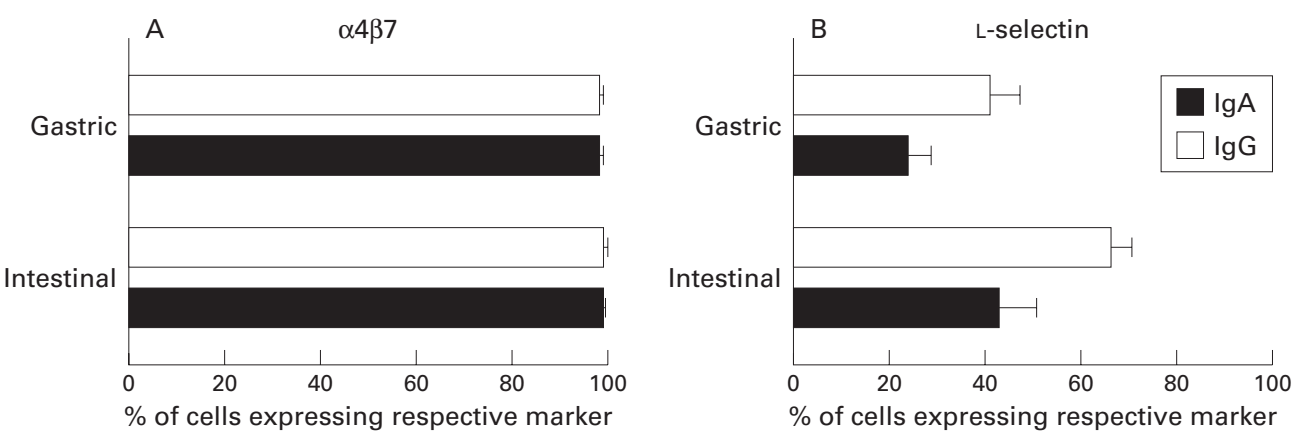

Figure 1 Homing receptor expression on cholera toxin B subunit (CTB) specific antibody secreting cells (ASC) induced by intestinal or gastric immunisations. Circulating lymphocytes were collected seven days after either intestinal or gastric immunisations and separated with regard to expression of $a 4 \beta 7(A)$ and L-selectin $(B)$, respectively. The frequencies of $C T B$ specific IgA and IgG secreting cells in the resulting cell populations were determined in ELISPOT assays, and expressed as the arithmetic mean (SEM) percentage of ASC expressing the respective markers.

whole $V$ cholerae organisms, using a combination of immunomagnetic cell sorting and ELISPOT techniques. We have previously used this technique to demonstrate substantial differences in homing receptor expression between circulating ASC induced by peroral and systemic immunisations. ${ }^{11}$ The volunteers participating in this part of the study were analysed for intestinal, gastric, and peripheral blood ASC frequencies, as reported in our accompanying paper. ${ }^{20}$ The two immunisation regimens induced ASC responses of similar magnitude comprising both IgA and IgG secreting cells. In this study, the vaccine specific ASC were used to examine homing receptor expression on $\mathrm{B}$ cells after activation by gastric and intestinal antigen delivery, respectively. Expression of L-selectin and $\alpha 4 \beta 7$ was similar on cells induced by both immunisation routes. Thus the mucosal homing receptor integrin $\alpha 4 \beta 7$ was expressed by almost all IgA and IgG ASC induced by intestinal immunisation whereas about half of these ASC coexpressed L-selectin (fig 1). Similarly, virtually all of the circulating CTB specific IgA and IgG ASC induced by gastric immunisation expressed $\alpha 4 \beta 7$, with only a smaller fraction of the ASC coexpressing L-selectin (fig 1).

Immunisations also induced circulating $\operatorname{IgA}$ and $\operatorname{IgM}$ secreting cells reacting with the whole cell component of the vaccine. The responder frequency was however much lower than with CTB specific ASC, and the frequencies of whole cell specific ASC were also lower than the frequencies of CTB specific ASC. Nevertheless, homing receptor expression was very similar on whole cell specific ASC induced by gastric and intestinal immunisation, regardless of the isotype produced, in that the majority of whole cell specific ASC resulting from both immunisation regimens were $\alpha 4 \beta 7+$ and L-selectin- (data not shown).

HOMING RECEPTOR EXPRESSION ON CIRCULATING H PYLORI SPECIFIC T CELLS

Circulating specific $\mathrm{T}$ cells are difficult to detect following mucosal immunisation, and we therefore decided to study $\mathrm{T}$ cell responses to the gastric antigen $H$ pylori MP and the systemic antigen TT in $H$ pylori infected cells. The proliferative response of $\mathrm{CD} 4+$ and $\mathrm{CD} 8+$ cells positive or negative for L-selectin or $\alpha 4 \beta 7$ was determined, after magnetic separation of the respective cell subsets and culture for five days with either $H$ pylori MP or TT. All of the different populations of CD8 $+\mathrm{T}$ cells proliferated poorly (SI $<3$ in all volunteers) when stimulated with either MP or TT, and no conclusive comparisons could be made between the populations. The CD4+ cell populations on the other hand responded to the different stimulations in a characteristic way. SI values obtained when stimulating CD $4+\alpha 4 \beta 7+$ cells with $H$ pylori MP were always higher then those obtained when stimulating the same number of CD $4+\alpha 4 \beta 7-$ cells $(\mathrm{p}<0.02)$. Conversely, higher responses to TT were found in the CD $4+\alpha 4 \beta 7-$ cells than in the CD $4+\alpha 4 \beta 7+$ cells $(p<0.05)$ (fig $2 \mathrm{~A})$. When expression of
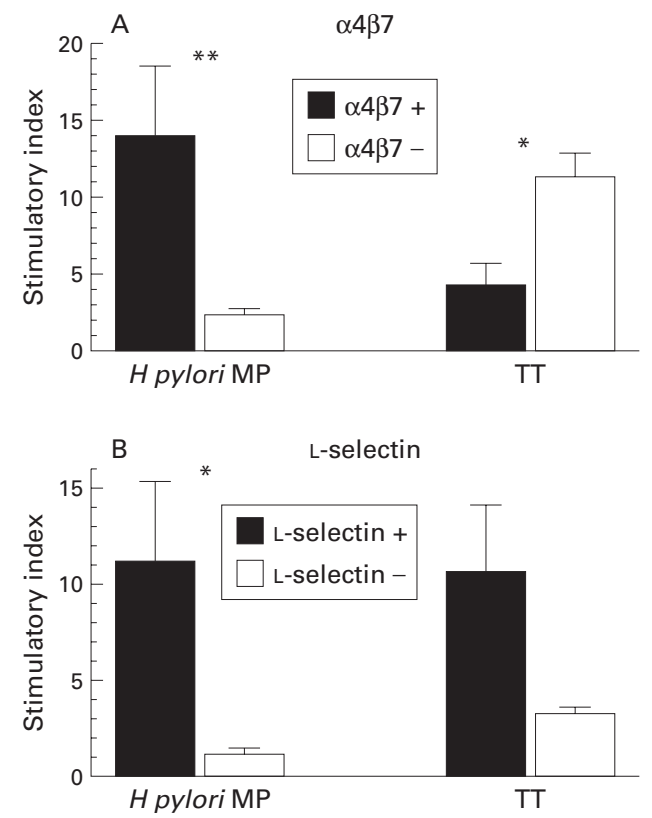

Figure 2 Homing receptor expression on Helicobacter pylori membrane protein (MP) and tetanus toxoid (TT) specific circulating CD $4+T$ cells. Circulating $T$ cells were collected from $H$ pylori infected individuals and $C D 4+$ cells isolated by incubation with CD4 specific magnetic beads. They were then further separated with regard to intergrin a $4 \beta 7$ (A) or L-selectin (B). Each of the cell subsets was stimulated with H pylori MP or TT, and proliferation assayed five days later. Data are expressed as arithmetic mean (SEM) of proliferative index in $\alpha 4 \beta 7+$ and $\alpha 4 \beta 7-$ cells in (A), and L-selectin + and L-selectin- cells in (B). ${ }^{\star} p<0.05,{ }^{\star} p<0.02$ for comparison between positive and negative cells stimulated with the respective antigens. 

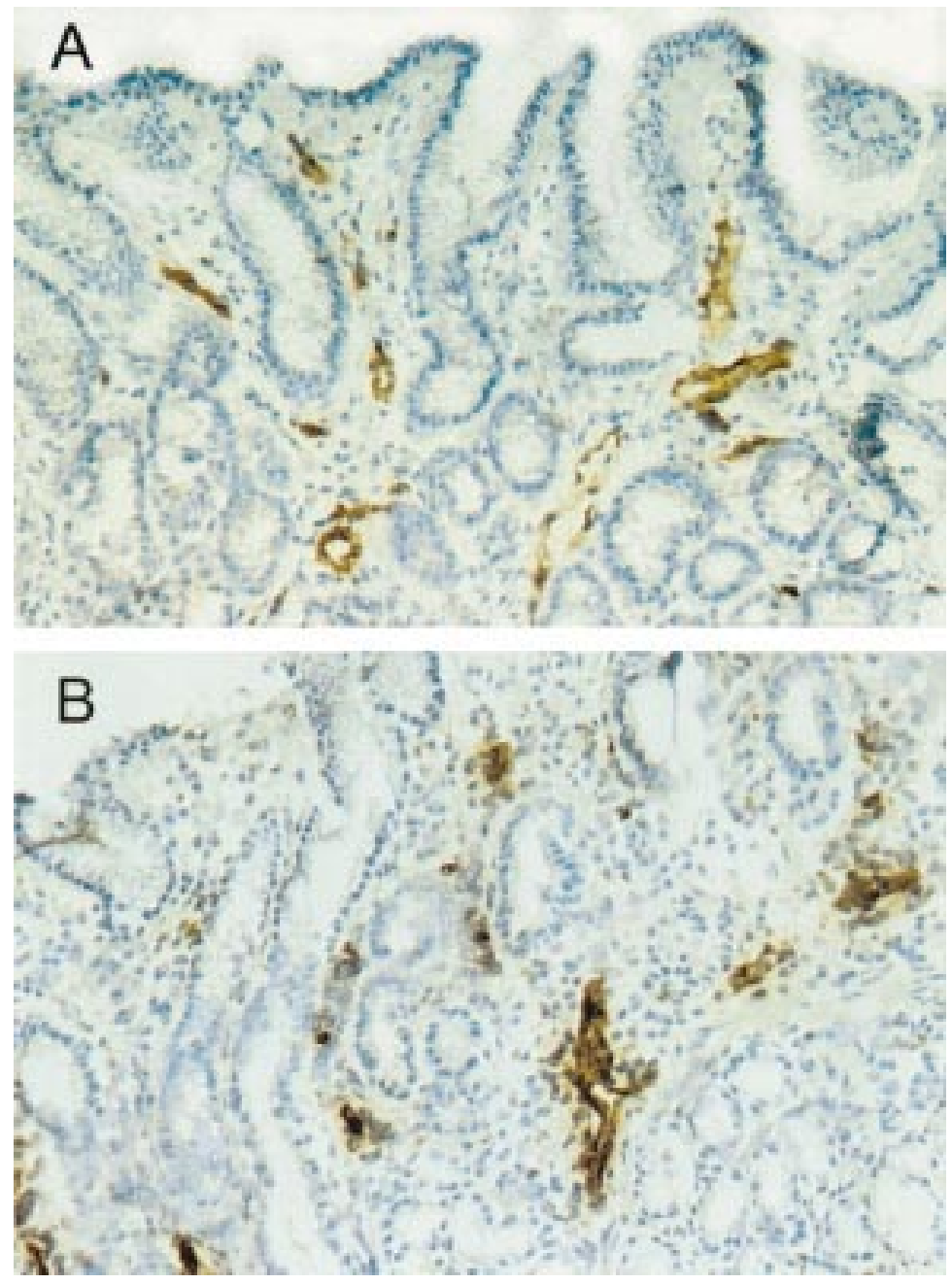

Figure 3 Mucosal addressin cellular adhesion molecule 1 (MAdCAM-1) expression in gastric mucosa. Gastric biopsies were collected from the antrum mucosa of uninfected $(A)$ and Helicobacter pylori infected (B) individuals, and MADCAM-1 expression on endothelial cells was determined by immunohistochemistry. Original magnification $\times 200$.

L-selectin was analysed, both $H$ pylori MP and TT reactive cells were found mainly in the CD4+L-selectin+ cells, and responses in the CD4+L-selectin- populations were much lower (fig 2B). Thus CD4+ $\mathrm{T}$ cells responding to $H$ pylori MP were found to be preferentially $\alpha 4 \beta 7+$ and L-selectin + , and those responding to TT were mainly $\alpha 4 \beta 7-$ and L-selectin+.

ENDOTHELIAL EXPRESSION OF MAdCAM-1 IN GASTRIC MUCOSA

Assessment of gastritis according to the updated Sydney system showed that the uninfected volunteers were free of gastritis while $H$ pylori infected individuals had a gastritis score of 1.9 (0.4). Immunhistochemical analysis of MAdCAM-1 expression by gastric endothelial cells revealed positive staining in the gastric mucosa of both $H$ pylori infected and uninfected individuals (fig 3). $H$ pylori infected individuals had 3.5 (1.8) (mean (SD)) MAdCAM-1+ vessels per $\mathrm{mm}^{2}$ of gastric mucosa. This value was not significantly different from that in uninfected volunteers (3.6 (1.8)). Furthermore, the total number of vessels, as determined by staining of von Willebrandt factor, was similar in the two groups of volunteers. The $H$ pylori infected volunteers had 4.5 (2.1) vessels per $\mathrm{mm}^{2}$ of gastric mucosa and uninfected individuals 4.5 (1.5) per $\mathrm{mm}^{2}$. Thus there was no difference in gastric MAdCAM-1 expression between $H$ pylori infected and uninfected individuals.

\section{Discussion}

In this study, we showed that both $\mathrm{B}$ and $\mathrm{T}$ lymphocytes activated by antigens present on the gastric mucosa expressed the mucosal homing receptor integrin $\alpha 4 \beta 7$. Furthermore, expression of L-selectin, which mediates homing to organised lymphoid tissues, was seen on most $H$ pylori reactive $\mathrm{T}$ cells and on about half of $\mathrm{B}$ cells induced by gastric immunisation. The endothelial ligand to $\alpha 4 \beta 7$, MAdCAM-1, was detected on gastric endothelial cells from both infected and uninfected individuals.

The existence of a common mucosal immune system has been convincingly demonstrated by studying migration of intestinally activated B cells to their final effector sites in the intestinal lamina propria and mucosa associated exocrine glands. ${ }^{26}{ }^{27}$ Several studies have indicated that mucosally activated $\mathrm{T}$ cells also migrate from their inductive site via the circulation to their final effector sites at mucosal surfaces. ${ }^{12}{ }^{1328}$ Nevertheless, there is a substantial degree of compartmentalisation within the mucosal immune system. ${ }^{29-31}$ Even within the intestinal tract, B cell responses induced by peroral and rectal antigen delivery are differentially distributed throughout the intestinal mucosa, and are strongest close to the site of the initial antigen encounter. ${ }^{32}$ As a prerequisite for the development of a vaccine against $H$ pylori infection, we have recently examined which immunisations can induce gastric immune responses. ${ }^{50}$ These studies showed that only $H$ pylori infected individuals had the capacity to mount gastric B cell responses after mucosal immunisations, and that both gastric and intestinal immunisations induced gastric as well as duodenal responses in these individuals. Thus $H$ pylori infected mucosa appears to be linked to the small intestine with regard to lymphocyte homing. In these experiments, we cannot rule out the possibility that some antigens from the gastric immunisations may have reached intestinal inductive sites. However, this is unlikely as vaccine administration through an endoscope made it possible to accurately deliver small droplets and ensure by inspection that the fluid was spread over the mucosa and absorbed almost instantly. Furthermore, the high binding capacity of CTB to ganglioside GM1, which is present on all nucleated cells, probably resulted in rapid capture of all CTB in the vaccine to the gastric mucosa.

To better understand the mechanisms behind gastric lymphocyte recruitment, we examined homing receptor expression by human B cells activated by antigens present in the stomach. Chronic $H$ pylori infection does not give rise to detectable numbers of circulating $B$ cells spontaneously secreting antibodies to $\mathrm{H}$ pylori antigens, ${ }^{4}$ and there is currently no $H$ pylori vaccine licenced for human use. Therefore, we 
chose to study B cell responses to two different types of well characterised mucosal antigens, the potent protein immunogen CTB as well as whole inactivated $V$ cholerae organisms. These studies showed that circulating ASC induced by gastric immunisations carry the same set of homing receptors as cells induced by intestinal immunisations, in that almost all cells express $\alpha 4 \beta 7$ and less than half coexpress L-selectin. The same set of homing receptors has previously been detected on B cells induced by peroral or rectal immunisation with the same cholera vaccine. ${ }^{11}$ These results suggest that $\mathrm{B}$ cells induced at any site along the gastrointestinal tract express the same set of homing receptors and therefore a putative human therapeutic vaccine against $H$ pylori infection could potentially be delivered to the small intestine and not directly to the gastric mucosa.

In contrast with $\mathrm{B}$ cells, circulating $\mathrm{T}$ cells specific for $H$ pylori antigens can be detected in the circulation of the majority of infected individuals. Circulating $\mathrm{T}$ cells specific for $\mathrm{H}$ pylori $\mathrm{MP}$ were found in the $\alpha 4 \beta 7+$ subpopulation. In previous studies, $T$ cells induced by the intestinal pathogen rotavirus or orally delivered antigens have also been shown to reside in the $\alpha 4 \beta 7+\mathrm{T}$ cell fraction. ${ }^{10}{ }^{13} 33$ Our results in the present study therefore reinforce the similarities in homing receptor usage between cells activated by intestinal or gastric antigens. Earlier studies have also shown that $\mathrm{T}$ cells induced by parenteral tetanus or mumps vaccination preferentially are $\alpha 4 \beta 7$ negative. ${ }^{10}{ }^{13}$ These results were confirmed in the present study, showing that TT specific T cells resulting from subcutaneous immunisations were mainly found in the $\alpha 4 \beta 7-$ subset. However, we also showed for the first time that parenteral immunisation with TT gave rise to specific $\mathrm{T}$ cells expressing L-selectin. Somewhat unexpectedly, most $H$ pylori reactive $\mathrm{T}$ cells were also found in the L-selectin+ fraction. Therefore, expression of L-selectin differs between $\mathrm{B}$ and $\mathrm{T}$ cells activated by gastric antigen delivery, in that $H$ pylori specific $\mathrm{T}$ cells were preferentially detected in the L-selectin+ fraction whereas $\mathrm{B}$ cells induced by gastric cholera vaccination were either L-selectin+ or L-selectin-. This may be explained by the assumption that $H$ pylori reactive $\mathrm{T}$ cells detected were probably memory cells whereas the vaccine specific ASC detected were effector cells. Different expression of L-selectin has been detected on so called central memory and effector memory $\mathrm{T}$ cells, in the sense that central memory cells express L-selectin while effector memory cells are L-selectin negative. ${ }^{34}$

In a recent study, Michetti and colleagues ${ }^{35}$ demonstrated that antibodies against integrin $\alpha 4 \beta 7$ blocked protection against $H$ felis infection, previously induced by oral immunisation with $H$ felis sonicate. Based on these findings, we examined if the increased lymphocyte migration to the $H$ pylori infected human mucosa was caused by increased expression of the mucosal addressin MAdCAM-1, the endothelial ligand to $\alpha 4 \beta 7$. This however was not the case as gastric mucosa from infected and uninfected individuals had almost identical expression of MAdCAM-1. Therefore, other mechanisms must be responsible for the increased lymphocyte migration to the human stomach in $H$ pylori infection. These could involve previously detected increased gastric expression of intercellular adhesion molecule 1 in $H$ pylori infected individuals. ${ }^{17}$ Increased local production of $\mathrm{B}$ cell attracting chemokine 1 (BCA-1), as well as interleukin 8, RANTES, and growth related oncogene $\alpha(\mathrm{GRO}-\alpha)$ has also been detected during $H$ pylori infection, ${ }^{36-38}$ and may also contribute to gastric lymphocyte accumulation.

In conclusion, in this study we have demonstrated that both $\mathrm{B}$ cells and $\mathrm{T}$ cells activated by antigens delivered to the gastric mucosa carry mucosal homing receptors, similar to those on cells activated in the intestine. This, together with the observation that gastric endothelial cells express MAdCAM-1, may partly explain the similar homing behaviour of cells activated in the stomach and small intestine. These observations also suggest that a potential future vaccine against $H$ pylori may not have to be targeted directly to the gastric mucosa but might be delivered to the small intestine.

We thank all volunteers who participated in this study. We are grateful to Dr Annika Hamlet for help with recruitment of the volunteers, and to Camilla Johansson and the staff at the gastroenterological department at Sahlgrenska University Hospital for their technical assistance and generous help. The generous supply of antibodies ACT-1 and 1AØ3 from Leukosite Inc. is gratefully acknowledged. This study was supported by the Swedish Medical Research Council (grant No 16X-13055), the Swedish Foundation for Strategic Research, the Swedish Medical Society, the Gothenburg Royal Science Foundation, and by a Society, the Gothenburg Royal Science Foundation, and by a setts, USA.

1 Parsonnet J, Hansen S, Rodriguez L, et al. Helicobacter pylori infection and gastric lymphoma. $N$ Engl $f$ Med 1994;330:1267-71.

2 Peek RM, Blaser MJ. Pathophysiology of Helicobacter pylori induced gastritis and peptic ulcer disease. Am F Med 1997; 102:200-7.

3 Genta RM, Gurer IE, Graham DY. Gastric lymphoid follicles in Helicobacter pylori infection: frequency, distribution, and response to triple therapy. Hum Pathol 1993;24: 577-83.

4 Mattsson A, Quiding-Järbrink $\mathrm{M}$, Lönroth $\mathrm{H}$, et al. Antibody-secreting cells in the stomach of symptomatic and asymptomatic Helicobacter pylori infected subjects. Infect Immun 1998;66:2705-12.

5 Mattsson A, Lönroth $\mathrm{H}$, Quiding-Järbrink $\mathrm{M}$, et al. Induction of B cell responses in the stomach of Helicobacter pylori-infected subjects after oral cholera vaccination. $f$ Clin Invest 1998;102:51-6.

6 Springer TA. Traffic signals for lymphocyte recirculation and leukocyte emigration: the multistep paradigm. Cell 1994;76:301-14.

7 Butcher EC, Picker LJ. Lymphocyte homing and homeosta-

sis. Science 1996;272:60-6.
8 Briskin M, Winsor-Hines D, Shyjan A, et al. Human mucosal addressin cell adhesion molecule-1 is preferentially expressed in intestinal tract and associated lymphoid tially expressed in intestinal tract and
tissues. Am F Pathol 1997;151:97-110.

9 Kantele JM, Arvilommi H, Kontiainen S, et al. Mucosally activated circulating human B cells in diarrhoea express homing receptors directing them back to the gut. Gastroenterology 1996;110:1061-7.

10 Rott LS, Rose JR, Bass D, et al. Expression of mucosal homing receptor $\alpha 4 \beta 7$ by circulating CD4+ cells with memory for intestinal rotavirus. F Clin Invest 1997;100:1204-8

11 Quiding-Järbrink $M$, Nordström I, Granströn G, et al. Differential expression of tissue-specific adhesion molecules on human circulating antibody-forming cells after systemic, enteric, and nasal immunization. A molecular basis for the compartmentalization of effector B cell responses. F Clin Invest 1997;99:1281-6.

12 Williams MB, Rose JR, Rott LS, et al. The memory B cell subset responsible for the secretory IgA response and protective humoral immunity to rotavirus expresses the intestinal homing receptor, $\alpha 4 \beta 7$. F Immunol 1998;161:422735.

13 Kantele A, Zivny J, Häkkinen M, et al. Differential homing commitments of antigen-specific $\mathrm{T}$ cells after oral or parenteral immunisation in humans. F Immunol 1999;162: 5173-7. 
14 Campbell JJ, Haraldsen G, Pan J, et al. The chemokine receptor CCR4 in vascular recognition by cutaneous but receptor CCR4 in vascular recognition by cutaneous but
not intestinal memory T cells. Nature 1999;400:776-80.

15 Zabel BA, Agace WW, Campbell JJ, et al. Human G proteincoupled receptor GPR-9-6/CC chemokine receptor 9 is selectively expressed on intestinal homing T lymphocytes, mucosa lymphocytes, and thymocytes and is required for thymus-expressed chemokine-mediated chemotaxis. $\mathcal{F}$ Exp Med 1999;190:1241-55.

16 Dogan A, Du M, Koulis A, et al. Expression of lymphocyte homing receptors and vascular addressins in low-grade gastric B-cell lymphomas of mucosa-associated lymphoid tissue. Am ₹ Pathol 1997;151:1361-9.

17 Hatz RA, Rieder G, Stolte M, et al. Pattern of adhesion molecule expression on vascular endothelium in Helicobacter pylori-associated antral gastritis. Gastroenterology 1997;112:1908-19.

18 Hamlet AK, Erlandsson KIM, Olbe L, et al. A simple, rapid and highly reliable capsule-based $14 \mathrm{C}$ urea breath test for diagnosis of $H$. pylori infection. Scand f Gastroenterol 1995; diagnosis of

19 Holmgren J, Svennerholm A-M, Jertborn M, et al. An oral B subunit: whole cell vaccine against cholera. Vaccine 1992:10:911-14

20 Quiding-Järbrink $\mathrm{M}$, Lönroth $\mathrm{H}$, Ahlstedt $\mathrm{I}$, et al. Human gastric B cell responses can be induced by intestinal immunisation. Gut 2001;49:512-8.

21 Dixon MF, Genta RM, Yardley JH, et al. Classification and grading of gastritis. The upgraded Sydney system. International Workshop on the Histopathology of Gastritis. Am F Surg Pathol 1996;20:1161-81.

22 Lakew M, Nordström I, Czerkinsky C, et al. Combined immunomagnetic cell separation and ELISPOT techniques define the phenotype of circulating antibodyforming cells. F Immunol Methods 1997;203:193-8.

23 Czerkinsky C, Moldoveanu Z, Mestecky J, et al. A novel two-colour ELISPOT assay. I: Simultaneous detection of distinct types of antibody-secreting cells. $\mathcal{F}$ Immunol distinct types of antib

24 Quiding $M$, Nordström I, Kilander A, et al. Intestinal immune responses in humans. Oral cholera vaccination induces strong intestinal antibody responses and interferon-gamma production and evokes local immunological memory. $\mathcal{F}$ Clin Invest 1991;88:143-8.

25 Wennerås C, Svennerholm A-M, Ahren C, et al. Antibodysecreting cells in human peripheral blood after oral immunisation with an inactivated enterotoxigenic Escherichia coli vaccine. Infect Immun 1992;60:2605-11.

26 Mestecky J. The common mucosal immune system and current strategies for induction of immune responses in external secretions. F Clin Immunol 1987;7:265-76.
27 McGhee JR, Mestecky J, Dertzbaugh MT, et al. The mucosal immune system: from fundamental concepts to vaccine development. Vaccine 1992;10:75-88.

28 Wennerås C, Svennerholm A-M, Czerkinsky C. Vaccinespecific $\mathrm{T}$ cells in human peripheral blood after oral immunisation with an inactivated enterotoxigenic Escherichia coli vaccine. Infect Immun 1994;62:874-9.

29 Quiding-Järbrink $M$, Granström G, Nordström I, et al. Induction of compartmentalised $\mathrm{B}$ cell responses in the human tonsils. Infect Immun 1995;63:853-7.

30 Eriksson K, Quiding-Järbrink M, Osek J, et al. Specificantibody-secreting cells in the rectums and genital tracts of nonhuman primates following vaccination. Infect Immun 1998;66:5889-96.

31 Haneberg B, Kendall D, Amerongen HM, et al. Induction of specific immunoglobulin $\mathrm{A}$ in the small intestine, colonrectum, and vagina measured by a new method for collection of secretions from local mucosal surfaces. Infect Immun 1994;62:15-23.

32 Eriksson K, Quiding-Järbrink M, Osek J, et al. Anatomical segmentation of the intestinal immune response in non-human primates: Oral and rectal immunisation induce differential homing of B cells to sites defined by their source of vascularisation. Infect Immun 1999;67: 6210-12.

33 Rose JR, Williams MB, Rott LS, et al. Expression of the mucosal homing receptor $\alpha 4 \beta 7$ correlates with the ability of CD8+ memory $\mathrm{T}$ cells to clear rotavirus infection. $\mathcal{F}$ Virol 1998;72:726-30.

34 Sallusto F, Lenig D, Förster R, et al. Two subsets of memory T lymphocytes with distinct homing potentials and effector functions. Nature 1999;401:708-12.

35 Michetti M, Kelly CP, Kraehenbuhl J-P, et al. Gastric mucosal $\alpha 4 \beta 7$-integrin-positive CD4 $\mathrm{T}$ lymphocytes and immune protection against Helicobacter infection in mice. Gastroenterology 2000;119:109-18.

36 Mazzucchelli L, Blaser A, Kappeler A, et al. BCA-1 is highly expressed in Helicobacter pylori-induced mucosa-associated lymphoid tissue and gastric lymphoma. F Clin Invest 1999; 104:R49-54.

37 Yamaoka Y, Kita M, Kodama T, et al. Expression of chemokine mRNA in gastric mucosa with Helicobacter pylori infection. Gastroenterology 1996;110:A1049.

38 Shimoyama T, Everett SM, Dixon MF, et al. Chemokine mRNA expression in gastric mucosa is associated with Helicobacter pylori cagA positivity and severity of gastritis. $\mathcal{f}$ Clin Pathol 1998;51:765-70. 